\title{
The benefits of hydrotherapy to patients with spinal cord injuries
}

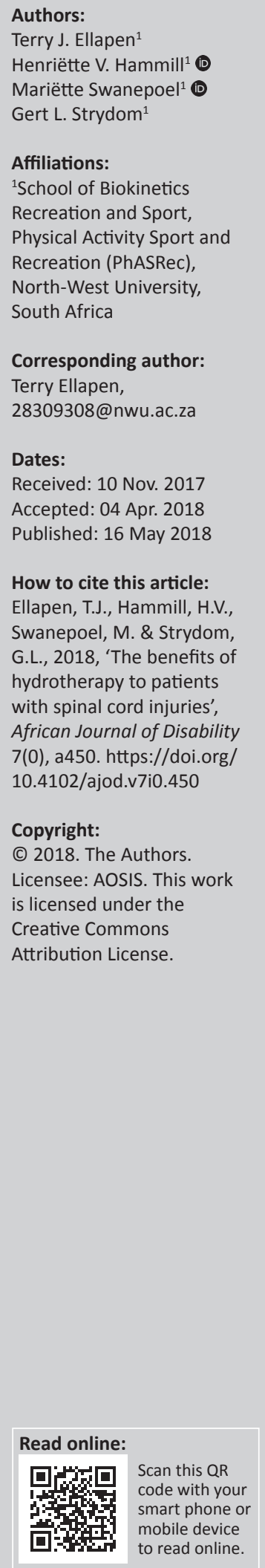

Authors:

Gert L. Strydom

Recreation and Sport,

Physical Activity Sport and

Recreation (PhASRec)

North-West University,

Terry Ellapen,

Dates:

Accepted: 04 Apr. 2018

Swanepoel, M. \& Strydom,

G.L., 2018, 'The benefits of

African Journal of Disability

7(0), a450. https://doi.org/

Copyright:

Licensee: AOSIS. This work

is licensed under the

Creative Commons

mobile device

to read online.
Background: Many patients with spinal cord injury (PWSCI) lead sedentary lifestyles, experiencing poor quality of life and medical challenges. PWSCI don't like to participate in land-based-exercises because it's tedious to perform the same exercises, decreasing their rehabilitative compliance and negatively impacting their well-being. An alternative exercise environment and exercises may alleviate boredom, enhancing compliance.

Objectives: Discuss the benefits of hydrotherapy to PWSCI concerning underwater gaitkinematics, thermoregulatory and cardiovascular responses and spasticity.

Methodology: A literature surveillance was conducted between 1998 and 2017, through the Crossref meta-database and Google Scholar, according to the PRISMA procedures. Key search words were water-therapy, aquatic-therapy, hydrotherapy, spinal cord injury, rehabilitation, human, kinematics, underwater gait, cardiorespiratory, thermoregulation and spasticity. The quality of each paper was evaluated using a modified Downs and Black Appraisal Scale. The participants were records pertaining to PWSCI and hydrotherapy. The outcomes of interest were: hydrotherapy interventions, the impact of hydrotherapy on gait-kinematics, thermoregulation during water submersion and cardiorespiratory function of PWSCI. Omitted records included: non-English publications from before 1998 or unrelated to hydrotherapy and PWSCI. The record screening admissibility was performed as follows: the title screen, the abstract screen and the full text screen.

Results: Literature search identified 1080 records. Upon application of the exclusion criteria, 92 titles, 29 abstracts and 17 full text records were eligible. Only 15 records were selected to be included in this clinical commentary. Evidence shows a paucity of randomised control trials (RCT) conducted in this field.

Conclusion: Hydrotherapy improves PWSCI underwater gait-kinematics, cardiorespiratory and thermoregulatory responses and reduces spasticity.

\section{Introduction}

Hydrotherapy, also known as aquatic or water therapy, has long been perceived as an effective, yet underutilised, therapeutic modality (Kesiktas et al. 2004). The benefits of hydrotherapy include enhanced aerobic capacity, improved muscle strength and endurance, increased joint range of motion (anti-spasticity), as well as decreased muscle fatigue and joint pain, enhanced cardiorespiratory functioning and a reduced cardiometabolic risk profile (Kesiktas et al. 2004). The majority of patients with spinal cord injury (PWSCI) lead sedentary lives, associated with a poor cardiometabolic profile (diabetes mellitus, increased insulin resistance, decreased insulin sensitivity, increased adiposity, obesity and body mass index as well as poor cardiorespiratory function) (Nooijen et al. 2016). Attempts to combat the poor cardiometabolic risk profile of PWSCI usually involve upper limb land-based exercises (Tweedy et al. 2016). La Fountaine et al. (2015) reported that these upper limb exercises are not as effective as lower limb exercises with regard to expending energy. A primary goal of exercising is to increase PWSCI's energy expenditure, thereby improving their poor cardiometabolic risk profile (La Fountaine et al. 2015). In order to increase energy expenditure, the rehabilitation programme's frequency, intensity and duration are increased, so that in turn it often leads to more upper limb injuries (Ellapen et al. 2017). Ellapen et al. (2017) reported that habitual use of the same upper body exercises leads to overuse orthopaedic injuries and boredom, resulting in poor rehabilitative exercise adherence. Strydom et al. (2009) reported that variation in habitual exercise and rehabilitative regimes increases patient adherence and subsequently is able to positively impact the realisation of the programme objectives. Hydrotherapy provides the following alternative options to land-based exercises: (1) a different rehabilitative environment, (2) the prescription of different upper limb and core exercises 
and (3) the opportunities for group and/or individual rehabilitation sessions with the exercise therapist (thereby increasing social interaction) (Kesiktas et al. 2004).

The therapeutic benefits of hydrotherapy relate to the following fundamental principles of hydrodynamics: (1) density, (2) drag, (3) buoyancy, (4) hydrostatic pressure and (5) thermodynamics. Density is explained using Archimedes' law of buoyancy, that the upward buoyant force exerted on an object immersed in water is equal to the weight of the water (or fluid) that the object displaces. This means that the human body, being of lower density than water, is subjected to a buoyant force (bringing the body to the surface) equal to the weight of the water that is displaced by the body's immersion (Becker 2009).

Thus, buoyancy occurs when a person is immersed in water, producing water displacement and progressively offloading the force of gravity on the immersed joints. By immersing the patient in water up to the cervical, thoracic (xiphoid process) and hip (pubic symphysis) joints, the therapist is able to offload $85 \%, 60 \%$ and $40 \%$, respectively, of the patient's individual body weight (gravity) that would normally weigh down on the submersed joints (Becker 2009). Buoyancy has great therapeutic value by allowing PWSCI to become mobile in the water without the resistance of gravity. The water becomes a dynamic fluid medium that allows PWSCI to safely, spontaneously and independently exercise and stabilises their lumbopelvic hip, thoracic and cervical muscles without relying on the use of their upper limbs in order to support their posture during the exercise, as is often the case during land-based exercises. This can be the key in the prevention of upper limb overuse injuries.

Drag force refers to the size of the internal resistive friction against movement in the fluid medium (water) (Poyhonen et al. 2000). The magnitude of drag increases as more force is exerted by the person, but is immediately neutralised (returning to zero) upon the cessation of movement, thereby providing accommodative hydro-resistance and thus preventing injuries in a similar manner to land-based isokinetic accommodative resistance (Poyhonen et al. 2000).

Hydrostatic pressure is the pressure exerted by the water during equilibrium at a given point during submersion, caused by gravity. Hydrostatic pressure is directly influenced by the density of water and by the depth of submersion. Hydrostatic pressure assists in the dissipation of oedema, in the gradual increase in joint range of motion and in combatting spasticity (Becker 2009).

Thermodynamics refers to water's ability to transfer heat. A significant therapeutic value of hydrotherapy depends on its ability to retain heat, as well as the transfer thereof. Fortunately, water is an efficient conductor, transferring heat 25 times faster than that of an equivalent volume of air (Bailey et al. 2007). Hydrotherapy can be used at a variety of temperatures: Ice water baths are often used post-training by athletes to reduce the effect of delayedonset muscle soreness, to promote the dissipation of inflammation and to quicken their recovery from training (Bailey et al. 2007). Warm water immersion decreases muscle pain, increases vasodilation and blood circulation, lowers heart rate and enhances thermoregulatory responses (Munguia-Izquierdo \& Legaz_Arrese 2007; Ingram et al. 2009). The temperatures of typical hydrotherapy pools range from $33.5{ }^{\circ} \mathrm{C}$ to $35.5{ }^{\circ} \mathrm{C}$ (Bailey et al. 2007). Heat transmission starts immediately upon initial water submersion primarily because the human body has a lower heat capacity than water (Bailey et al. 2007).

Over and above the benefits regarding heat conduction, water has further benefits related to respiration: Boyle's law suggests that the volume of any gas varies inversely with the pressure exerted upon it. Greater submersion depth, therefore, increases the hydrostatic pressure against the thoracic cage, thereby inversely impacting its lung volume. The therapeutic benefit is the increased respiratory cost during water submersion, which expends more calories and improves respiratory efficiency, positively impacting one's cardiometabolic profile (Becker 2009).

According to the authors' knowledge, there have been two reviews published on aquatic therapy in relation to PWSCI (Li, Khoo \& Adan 2017; Recio, Stiens \& Kubrova 2017). Li et al. (2017) is the only systematic review that evaluates the quality of the research of aquatic therapy and exercise prescribed to PWSCI. However, Li et al. (2017) did not discuss the rehabilitation and exercise physiology mechanisms, but highlights the value of hydrotherapy. Recio et al. (2017) only describe the clinical anti-spasticity and ventilatory benefits of hydrotherapy for PWSCI; they neither describe the methodology used to find the papers nor the PWSCI's underwater gait kinematics, thermoregulatory and cardiorespiratory responses to aquatic therapy. This commentary combines the elements of rigorous methodology undertaken by Li et al. (2017) in their systematic review and the discussion of the therapeutic benefits of hydrotherapy for PWSCI. Further, the novelty of this commentary lies in the biomechanical discussion of hydrotherapy, specifically considering PWSCI's gait kinematics, cardiorespiratory, spasticity and thermoregulatory responses. The aim of this commentary is to determine the effect of hydrotherapy on PWSCI's gait kinematics, muscle spasticity, cardiorespiratory and thermoregulatory responses.

\section{Methods}

The authors followed the standard practices for systematic reviews: Preferred Reporting Items for Systematic Reviews and Meta-Analyses (PRISMA).

Information sources and searches: A literature search of peer-reviewed records was conducted using the following search engine: Crossref meta-database, which is an academic database comprising of the following search engines: 


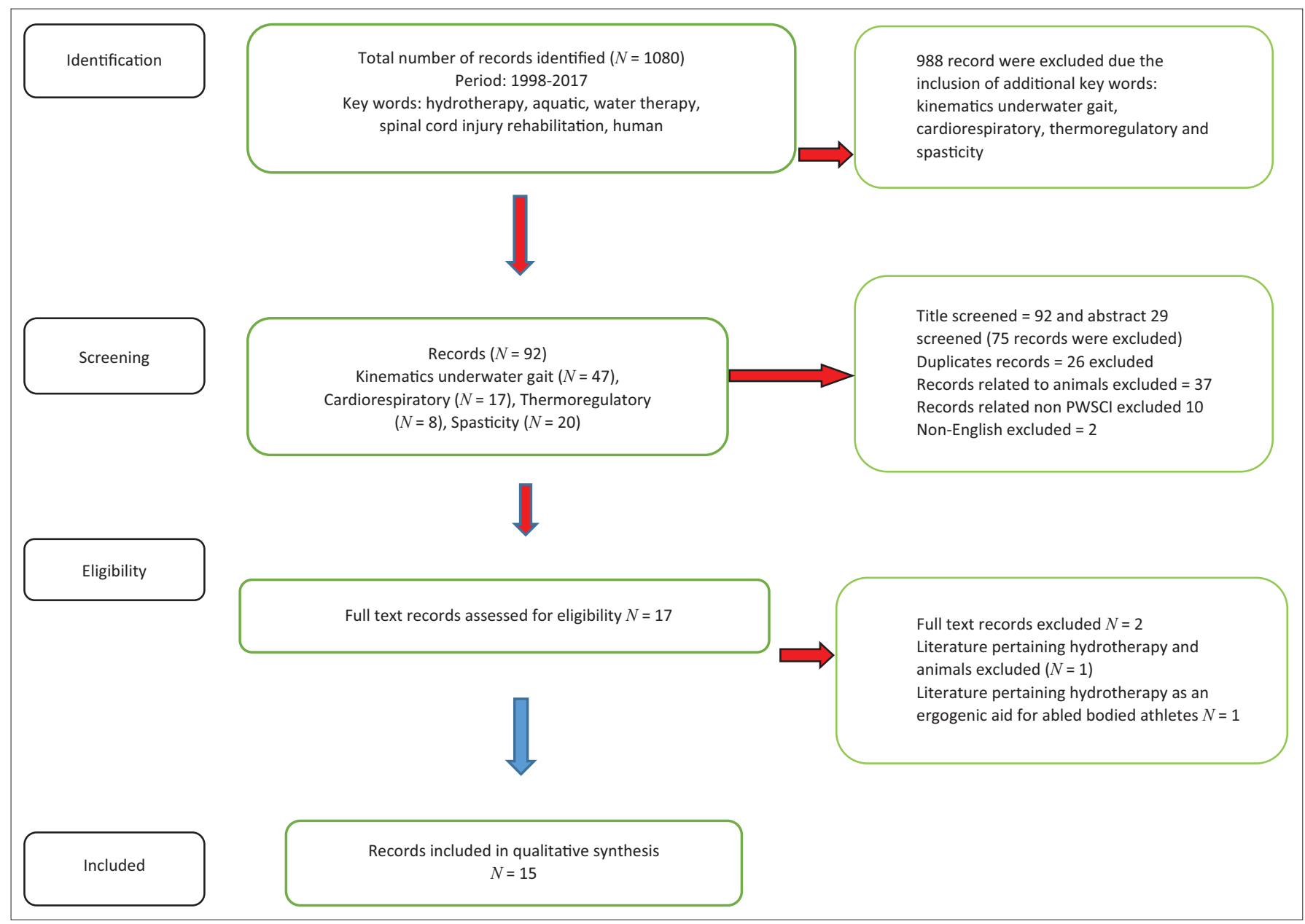

$N$, number of records; PWSCl, patients with spinal cord injuries.

FIGURE 1: Flow chart of the review process.

PubMed, Medline, Science Direct, Ebscohost, CINAHL and Google Scholar (Figure 1). The keywords used in the literature search were water therapy, aquatic therapy, hydrotherapy, spinal cord injury, rehabilitation, human, kinematics, underwater gait, cardiorespiratory, thermoregulation and spasticity. The screening eligibility of records was performed in the following three steps: (1) the title screen, (2) the abstract screen and (3) the full text screen. The records were screened by TJE, HVH, MS and GLS.

Eligibility criteria: The participants in this study were records pertaining to PWSCI and hydrotherapy; the intervention was not necessarily a therapeutic intervention but is interpreted as an exposure, namely the effect of hydrotherapy on the wellbeing of PWSCI. The outcomes of interest were (1) hydrotherapy interventions for PWSCI, (2) the impact of hydrotherapy on PWSCI gait kinematics, (3) the effect of hydrotherapy on PWSCI thermoregulation during water submersion and (4) the impact of hydrotherapy on PWSCI cardiorespiratory function. The exclusion criteria were (1) publications prior to 1998, (2) literature pertaining to hydrotherapy and animals, (3) literature related to hydrotherapy as an ergogenic aid among ablebodied athletes, (4) the impact of hydrotherapy on the health and well-being of able-bodied athletes and (5) non-English papers.

\section{Study selection: The appraisal of the quality of records}

All records were filtered based on the appropriateness of their title and the inclusion criteria. The quality of each record was appraised using a modified Downs and Black Appraisal Scale, which examines the quality of randomised controlled trials and non-randomised papers (Downs \& Black 1998) (Table 1). The evaluation of the quality of each record reduced the risk of researcher biasness. The modified Downs and Black checklist was adopted as not all the items on the original checklist were related to this paper, as similarly cited in Ellapen et al. (2017). The modified checklist comprises 16 questions with a maximum of 16 points. Answers were given a score of either 0 (no) or 1 (yes). The questions adopted from the Downs and Black Appraisal Scale were questions number $1,3,4,5,6,10,11,12,13,14,18,20,21,22,23$ and 27. These questions are categorised into four sections in order to assess the overall quality of each paper (Table 2). The sections include reporting prowess ( $n=5$ questions), external validity ( $n=3$ questions), internal validity ( $n=3$ questions) and power of significance ( $n=5$ questions) (Downs \& Blacks 1998). All authors were allowed to dispute the scoring of each record. The authors would then discuss scores and adopted the mutually accepted score. The sum of these scores was then 
TABLE 1: Appraisal of records according to the modified Downs and Black Appraisal Scale.

\begin{tabular}{lcc}
\hline Authors & $\begin{array}{c}\text { Reporting } \\
(\boldsymbol{n}=\mathbf{5})\end{array}$ & $\begin{array}{c}\text { External validity } \\
(\boldsymbol{n}=\mathbf{3})\end{array}$ \\
\hline Zamparo and Pagliaro (1998) & 5 & 2 \\
Gass and Gass (2001) & 5 & 2 \\
Gass, Gass and Pitetti (2002) & 5 & 2 \\
Kesiktas et al. (2004) & 5 & 2 \\
\hline Prosser (2007) & 4 & 3 \\
Becker (2009) & 4 & 1 \\
Lucksch et al. (2013) & 5 & 3 \\
Tamburella et al. (2013) & 5 & 2 \\
Jung et al. (2014) & 5 & 3 \\
Stevens et al. (2014) & 5 & 2 \\
Stevens and Morgan (2015) & 5 & 2 \\
Tweedy et al. (2016) & 4 & 1 \\
Li et al. (2017) & 4 & 0 \\
Recio et al. (2017) & 4 & 0 \\
Wall, Falvo and Kesten (2017) & 5 & 3 \\
Overall rating & 4.6 & 1.3 \\
\hline
\end{tabular}

Downs and Black Appraisal

$N$, number; $\mathrm{x}$, sum of Downs and black appraisal.

converted to a percentage so as to rate the overall quality of the individual papers (Downs \& Black 1998). The overall quality of the papers was graded using a scale defined as follows: < 50\% (weak), 50\% - 69\% (fair), 70\% - 79\% (good) and $<80 \%$ (very good) (Downs \& Black 1998).

\section{Results}

The literature review identified 1080 records by the use of the key search words (water therapy, aquatic therapy, hydrotherapy, spinal cord injury, rehabilitation and human). The application of additional key words (kinematics underwater gait, cardiorespiratory, thermoregulation and spasticity) resulted in 92 records. All the titles of each record were screened ( $n=92)$; however, only 29 abstracts were screened. Thirty-seven animal records, 26 duplicate records, 10 records pertaining to non-PWSCI and 2 non-English records were excluded. The remaining 17 full text records were reviewed. Two full text records comprised of one animal record and the other that pertained to the adoption of hydrotherapy as an ergogenic aid. The remaining 15 records comprised of 4 systematic reviews pertaining to PWSCI (but 2 specific to hydrotherapy), 7 non-randomised control trials, 2 randomised control and 2 case studies (Table 2). Table 1 assesses the quality of each record according to the modified Downs and Black Appraisal Scale (in an attempt to eliminate risk of biasness). A descriptive overview of the characteristics and findings of the studies is found in Table 2. A total of 142 participants were reported (but 83 PWSCI), with sample sizes varying from 1 to 30 and participant age varying from 5 to 70 years. Five studies provided kinanthropometric characteristics, whereas 5 studies considered the number of years injured, and 10 studies described the aquatic exercise intervention. The overall quality of the studies was rated as fair $(62.0 \%)$ (Table 1$)$.

\section{Discussion}

The discussion will focus on the empirical findings of the impact of hydrotherapy on the gait kinematics of
PWSCI as well as their thermoregulatory, spasticity and cardiorespiratory responses.

\section{Kinematic gait analyses}

The kinematic gait analysis studies involved the review of the form or technique of PWSCI underwater walking. Zamparo and Pagliaro (1998), Prosser (2007) and Tamburella et al. (2013) all concur that the patients' gait kinematics, walking speed and stride length improved after the completion of hydrotherapy. However, the aforementioned authors failed to describe the biomechanical mechanism facilitating PWSCI-enhanced gait kinematics. It was postulated that hydrostatic pressure combined with the effects of buoyancy enhanced the patients' lumbopelvic hip complex form and force closure, thereby enhancing their underwater gait kinematics. Buoyancy helped elevate the contralateral hip during the stance phase, thereby decreasing the muscle contraction force required to elevate the contralateral hip. Furthermore, buoyancy negated the effects of gravity, enhancing the swing phase of the ipsilateral hip. Zamparo and Pagliaro (1998) also reported that PWSCI energy expenditure was lower during underwater walking as compared to land-based walking at specific speeds, which allowed them to walk for longer. However, Zamparo and Pagliaro's (1998) study was limited by their research design (experimental, non-RCT and without concurrent controls). Therefore, Zamparo and Pagliaro (1998) recommended future empirical investigations adopting experimental RCT procedures with comparative concurrent controls in order to validate the findings of the prospective studies. However, Lucksch et al. (2013) and Jung et al. (2014) heeded to Zamparo and Pagliaro's (1998) recommendations. This computes into two RCT out of 92 records (2.1\%) published during the period of 1998 to 2017 pertaining to human PWSCI and hydrotherapy. The authors of this article strongly encourage more RCT examining the effects of hydrotherapy on PWSCI needs to be completed and published. This empirical evidence will help to encourage exercise therapists to prescribe hydrotherapy as 


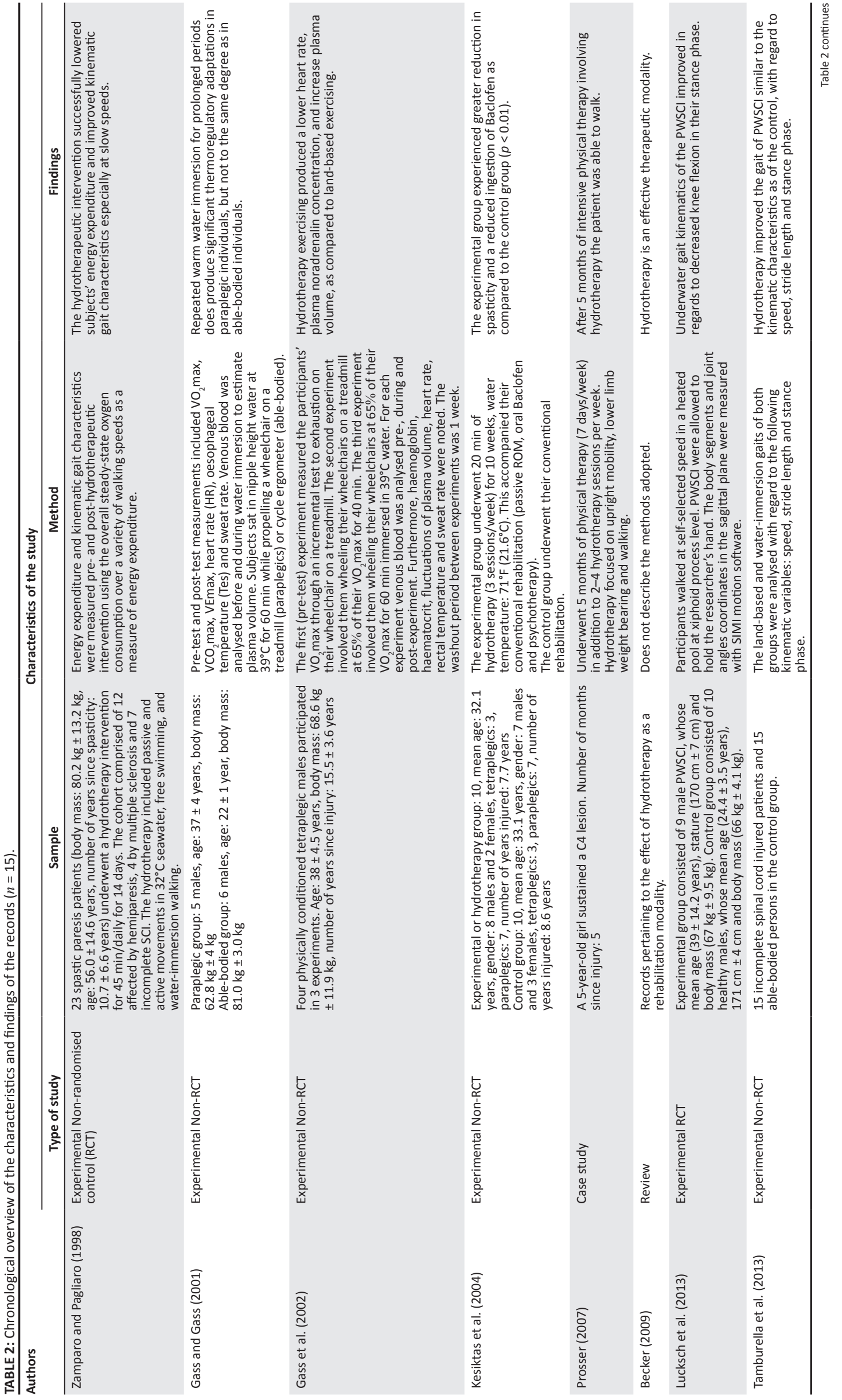




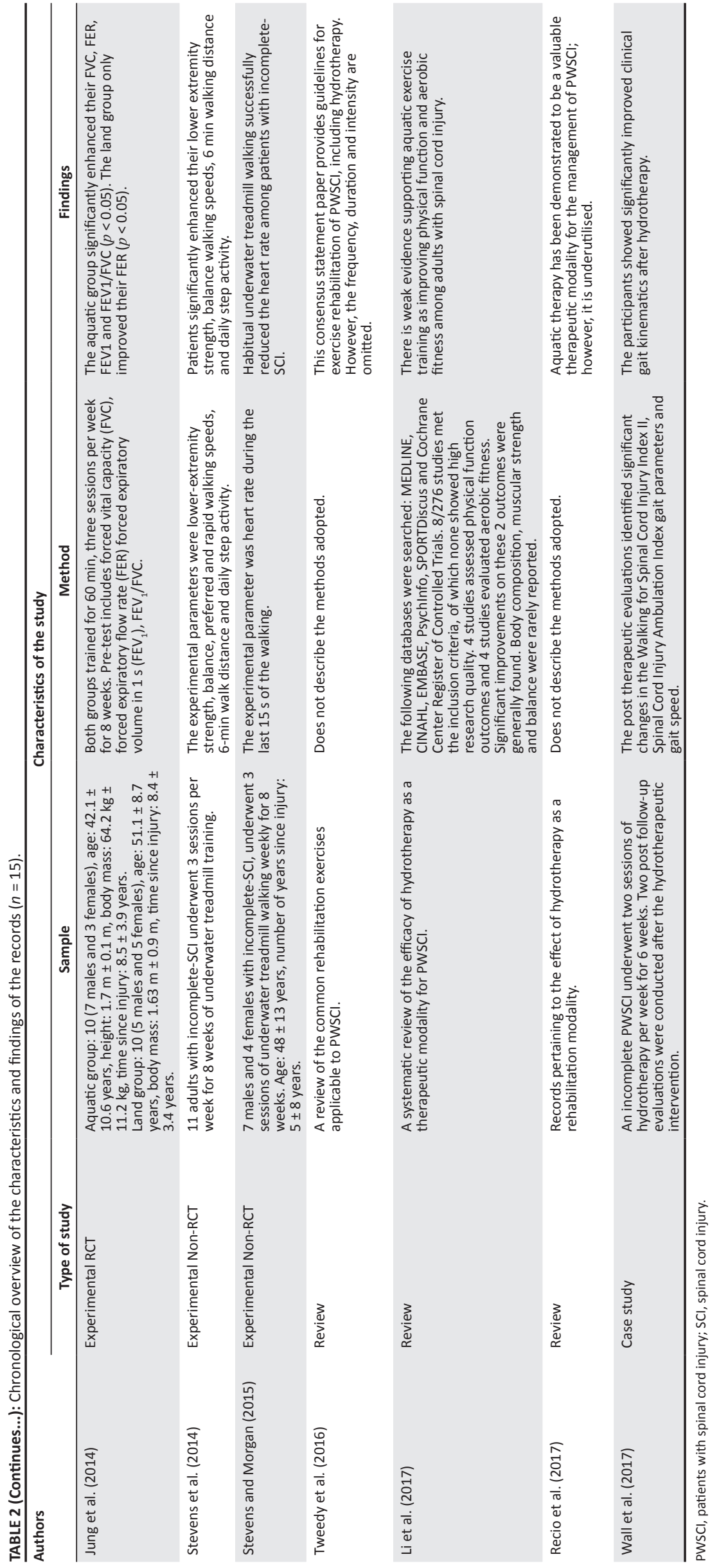


a supplement to other management practices of PWSCI. Gass and Gass (2001) and Gass et al. (2002) confirmed Zamparo and Pagliaro's (1998) postulation that increased exercise duration will augment energy expenditure of paraplegic patients in a manner that will positively impact their cardiometabolic profile. Tweedy et al. (2016) and Ellapen et al. (2017) both concur that lower limb exercises (i.e. walking and strengthening) expend more energy than upper limb exercises. Therefore, lower limb exercises are needed to increase PWSCI energy expenditure to help improve their cardiometabolic risk profile. The authors of this study recommend that PWSCI should engage in hydrotherapeutic walking before land walking. The hydrotherapeutic walking will serve to condition the PWSCI lower limb neuromuscular system, preparing them for land walking and simultaneously increasing energy expenditure, lowering their cardiometabolic risk profile.

\section{Thermoregulatory response to exercising in warm water submersion}

Exercising while submerged in warm water lowers the heart rate and enhances thermoregulatory responses, thereby prolonging the PWSCI's ability to exercise and thus increasing their aerobic capacity (Gass \& Gass 2001; Gass et al. 2002). The prolonged exercising during water submersion increases the patient's energy expenditure, thereby lowering their cardiometabolic risk profile. Water is an excellent conductor of heat, which enhances patients' ability to effectively thermoregulate their bodies when exercising and maintains a low core temperature (Becker 2009). This further physiological adaptation also contributes to the ability of patients to exercise for longer, thereby improving their cardiorespiratory function and energy expenditure (Gass et al. 2002; Becker 2009).

\section{Decreased spasticity response to hydrotherapy}

Kesiktas et al. (2004) reported that PWSCI experienced a significant reduction in muscle spasticity with a reduced dosage of oral baclofen because of hydrotherapy. This is the only study, among numerous clinical reports of reduced muscle soreness, spasticity and increased joint range of motion among arthritic patients, who also experience spasticity (Eversden et al. 2007), to report this result. Becker (2009) reported that the physiological rationale behind the efficacy of hydrotherapy on spasticity, muscle soreness and joint range of motion is enigmatic. Therefore, further clinical investigation should be undertaken so as to unravel the physiological mechanism of the efficacy of hydrotherapy in relation to the aforementioned maladies.

\section{Cardiorespiratory benefits}

The patient's cardiorespiratory adaptations are based on Boyle's law. When a person is submersed in water, the hydrostatic pressure against the body increases, thereby decreasing their lung volume (Becker 2009). Greater submersion depth increases the hydrostatic pressure, making breathing more costly. Becker (2009) reported that the patient's vital capacity is reduced by $6 \%-9 \%$ because of compression by external hydrostatic pressure which counteracts inspiratory muscle action. Energy expenditure at rest increases by $60 \%$ during neck-level submersion which in turn enhances inspiratory muscle strength and endurance, serving as an effective respiratory rehabilitative exercise medium able to counteract respiratory diseases (Taylor \& Morrison 1999; Becker 2009). Pachalski and Mekraski (1980) reported that PWSCI gained a greater cardiorespiratory fitness improvement by following an aquatic exercise programme as compared to land-based exercises. Van Houtte, Vanlandewijck and Gosselink (2006) and Jung et al. (2014) reported that respiratory muscle rehabilitation conditioning programmes increase the expiratory muscle strength, vital capacity and residual volumes of PWSCI.

When a person is submerged in water, blood is displaced towards the heart, thereby enhancing central venous return, which in turn increases arterial and ventricular filling and results in a subsequent decrease in heart rate (Becker 2009). There is a significant increase in end-diastolic volume, producing a larger stroke volume. During aquatic exercising, maximal oxygen consumption is greater than that of land-based exercise, allowing for greater energy expenditure at slower speeds and prolonged activity (Becker 2009). During neck-level submersion, there is a decrease in sympathetic nervous activity which reduces peripheral resistances, thereby allowing greater venous return (Becker 2009). Stevens and Morgan (2015) reported that habitual underwater treadmill walking reduces PWSCI heart rate, suggesting enhanced cardiorespiratory function. It is hypothesised that the lower exercise heart rate experienced during underwater walking combined with effective thermoregulatory response will increase exercise duration or walking distance, thereby increasing energy expenditure, which in turn will positively impact on the cardiometabolic profile of PWSCI. It should, however, be noted that these suppositions require clinical validation.

\section{Conclusion}

Hydrotherapy aids in reducing PWSCI muscle spasticity and cardiometabolic risk profiles, while favourably enhancing underwater gait kinematics and cardiorespiratory capacity. However, more RCT should be undertaken to increase the present body of knowledge.

\section{Acknowledgements}

The authors would like to acknowledge the paper by Ellapen et al. (2017) that reviewed common upper limb injuries among wheelchair users, which led to the drafting of this article, which prescribes hydrotherapy as an alternative to traditional land-based exercise.

\section{Competing interests}

The authors declare that they have no financial or personal relationships that may have inappropriately influenced them in writing this article. 


\section{Authors' contributions}

T.J.E., H.V.H., M.S. and G.L.S. were responsible for conceptualising the article, literature search and drafting of the manuscript. T.J.E. and H.V.H. were responsible for revising the manuscript.

\section{References}

Bailey, D.M., Erith, S.J., Griffin, P.J, Doeson, A., Brewer, S.D., Gant, N. et al., 2007 Influence of cold-water immersion on indices of muscle damage following prolonged intermittent shuttle running', Journal of Sports Science 25(11), 1163prolonged intermittent shuttle running', Journal of $S$ Por
1170. https://doi.org/10.1080/02640410600982659

Becker, B.E., 2009, 'Aquatic therapy: Scientific foundations and clinical rehabilitation applications', Clinical Review: Current Concepts 1(9), 859-872. https://doi.org/ 10.1016/j.pmrj.2009.05.017

Downs, S.H. \& Black, N., 1998, 'The feasibility of creating a checklist for the assessment of the methodological quality both of randomized and non-randomized studies of health care interventions', Journal of Epidemiology in Community Health 52(6), 377-384. https://doi.org/10.1136/jech.52.6.377

Ellapen, T.J., Swanepoel, M., Hammill, H.V. \& Strydom, G.L., 2017, 'The health benefits and constraints of exercise therapy for wheelchair users: A clinical commentary', African Journal of Disability 6, a337. https://doi.org/10.4102/ajod.v6i0.337

Eversden, L., Maggs, F., Nightingale, P. \& Jobanputra, P., 2007, 'A pragmatic randomised controlled trial of hydrotherapy and land exercises on overall well-being and quality of life in rheumatoid arthritis', BMC Musculoskeletal Disorders 8(1), 23. https://doi.org/10.1186/1471-2474-8-23

Gass, E.M. \& Gass, G.C., 2001, 'Thermoregulatory response to repeated warm wate immersion in subjects who are paraplegics', Spinal Cord 39(3), 149-155. https:// doi.org/10.1038/sj.sc.3101117

Gass, E.M., Gass, G.C. \& Pitetti, K., 2002, 'Thermoregulatory responses to exercise and warm water immersion in physically trained men with tetraplegia', Spinal Cord 40(9), 474-480. https://doi.org/10.1038/sj.sc.3101341

Ingram, J., Dawson, B., Goodman, C., Wallman, K. \& Beilby, J., 2009, 'Effect of water immersion methods on post exercise recovery from simulated team sport exercise', Journal of Science and Medicine in Sport 12(3), 417-421. https://doi. org/10.1016/j.jsams.2007.12.011

Jung, J., Chung, E., Kim, K., Lee, B.H. \& Lee, J., 2014. 'The effects of aquatic exercise on pulmonary function in patients with spinal cord injury', Journal of Physical Therapy and Science 26(5), 707-709. https://doi.org/10.1589/jpts.26.707

Kesiktas, N., Paker, N., Erdogan, N., Gulsen, G., Bicki, D. \& Yilmaz, H., 2004, 'The use of hydrotherapy for the management of spasticity', The American Society of Neurorehabilitation and Neural Repair 18(4), 268-273. https://doi.org/10.1177/ 1545968304270002

La Fountaine, M.F., Cirringliaro, C.M., Emmons, R.R., Kirshblum, S.C., Galea, M. Spungen, A.M. et al., 2015, 'Lipoprotein heterogeneity in persons with spinal cord injury: A model of prolonged sitting and restricted physical activity' cord injury: A model of prolonged sitting and restricted physical activity', Lipids in

ii, C., Khoo, S. \& Adnan, A., 2017, 'Effects of aquatic exercise on physical function and fitness among people with spinal cord injury: A systematic review', Medicine 96(11), e6328. https://doi.org/10.1097/MD.0000000000006328
Lucksch, D.D., Isreal, V.L., Ribas, D.R. \& Manffra, E.F., 2013, 'Gait characteristics of persons with incomplete spinal cord injury in shallow water', Journal of persons with incomplete spinal cord injury in shallow water', Journal of
Rehabilitation and Medicine 45, 860-865. https://doi.org/10.2340/16501977-1193

Munguia-Izquierdo, D. \& Legaz_Arrese, A., 2007, 'Exercise in warm water decreases pain and improves cognitive function in middle-aged women with fibromyalgia', Clinical and Experimental Rheumatology 25(6), 823-830.

Nooijen, C.J., Stam, H.J., Bergen, M.P., Bongers-Jassen, M.H., Valent, J., Van Langeveld, S. et al., 2016, 'Behavioural intervention increases physical activity in people with subacute spinal cord injury: A randomised trial', Journal of Physiotherapy 62(1) 35-41. https://doi.org/10.1016/j.jphys.2015.11.003

Pachalski, A. \& Mekraski, T., 1980, 'Effects of swimming on increasing of cardiorespiratory capacity in paraplegics', Paraplegia 18(3), 190-196. https://doi. org/10.1038/sc.1980.33

Poyhonen, T., Keshinen, K.L., Hautala, A. \& Malkia, E., 2000, 'Determination of hydrodynamic drag forces and drag coefficients on human leg/foot model during knee exercise', Clinical Biomechanics 15(4), 256-260. https://doi.org/10.1016/ S0268-0033(99)00070-4

Prosser, L.A., 2007, 'Locomotor training within an inpatient rehabilitation program after paediatric incomplete spinal cord injury', Physical Therapy 87(9), 1224-1232. https://doi.org/10.2522/ptj.20060252

Recio, A.C., Stiens, S.A. \& Kubrova, E., 2017, 'Aquatic-based therapy in spinal cord injury rehabilitation: Effective yet underutilized', Current Physical Medicine and Rehabilitation Reports 5(3), 108-112. https://doi.org/10.1007/s40141-017-0158-5

Stevens, S.L., Caputo, J.L., Fuller, D.K. \& Morgan, D.W., 2014, 'Effects of underwater treadmill training on leg strength, balance and walking performance in adults with incomplete spinal cord injury', Journal of Spinal Cord Medicine 38(1), 91-101. https://doi.org/10.1179/2045772314Y.0000000217

Stevens, S.L. \& Morgan, D.W., 2015,' Heart rate response during underwater treadmill training in adults with incomplete spinal cord injury. Topics in spinal cord injury rehabilitation, 2015 winter', Journal of Spinal Cord Medicine 21(1), 40-48.

Strydom, G.L., Wilders, C.J., Moss, S.J. \& Bruwer, E., 2009, 'A conceptual framework of Biokinetic procedures and referral system: An integrated protocol for the various health paradigms', African Journal for Physical, Health Education, Recreation and Dance 15(4), 641-649.

Tamburella, F., Scivoletto, G., Cosentino, E. \& Molinari, M., 2013, 'Walking in water and land after an incomplete spinal cord injury', American Journal of Physical and Medical Rehabilitation 92(10), e4-e15. https://doi.org/10.1097/PHM.0b013 e3182a1e6c3

Taylor, N.A. \& Morrison, J.B., 1999, 'Static respiratory muscle work during immersion with positive and negative respiratory loading', Journal of Applied Physiology 87(4), 1397-1403. https://doi.org/10.1152/jappl.1999.87.4.1397

Tweedy, S.M., Beckman, E.M., Geraghty, T.J., Theisen, D., Perret, C., Harvey, L.A. et al., 2016, 'Exercise and sports science Australia (ESSA) position statement on exercise and spinal cord injury', Journal of Science and Medicine in Sport 20(2), 108-115. https://doi.org/10.1016/j.jsams.2016.02.001

Van Houtte, S., Vanlandewijck, Y. \& Gosselink, R., 2006, 'Respiratory muscle training in persons with spinal cord injury: A systematic review', Respiratory Medicine 100(11), 1886-1895. https://doi.org/10.1016/j.rmed.2006.02.029

Wall, T., Falvo, L. \& Kesten, A., 2017, 'Activity specific aquatic therapy targeting gait for a patient with incomplete spinal cord injury', Physiotherapy Theory and Practice 33(4), 331-344. https://doi.org/10.1080/09593985.2017.1302026

Zamparo, P. \& Pagliaro, P., 1998, 'The energy cost of level walking before and after hydro-kinesi therapy in patients with spastic paresis', Scandinavian Journal of Medicine in Science and Sport 8(4), 228-28. https://doi.org/10.1111/j.16000838.1998.tb00196.x 